\title{
Receptor Status Accuracy in Breast Cancer: Core Biopsy or Excision? A Mini Review
}

\author{
Mohammed J Alyousef*, Mohammed M Hajla and Maha Abdel Hadi \\ Departments of Pathology and Surgery, Imam Abdulrahman Bin Faisal University, Saudi Arabia
}

*Corresponding author: Mohammed J Alyousef, Departments of Pathology and Surgery,Imam Abdulrahman Bin Faisal University, Saudi Arabia

\begin{abstract}
Aim

With the advent of minimally invasive surgical techniques core needle biopsy (CNB) has emerged as an accurate method of obtaining representative tissue providing both prognostic and diagnostic valuable information the management of breast cancer. This review aims to compare the precision and limitations in the reporting of CNB and excisional biopsies. (EB)

Materials and Methods: All patients diagnosed with breast cancer were reviewed. Review of the histological data was the essence of this study. Only patients whose receptors were tested in both core and excisions were included. The hormonal status of estrogen receptors (ER), progesterone receptors (PgR) and Her2 were evaluated immunohistochemically. Equivocal HER2 status was reassessed using Fluorescence in situ hybridization (FISH) test.

Results: The total number of patients diagnosed with breast cancer was 112. In $36(32.1 \%)$ patients complete synchronized immunohistological testing for both CNB and EB was executed and compared. The concordance rate observed in was as follows:ER was matched in 27 (75\%), PgR in 22 (61.1\%) Her2 in 34(94\%).The calculated concordance rate was 86\%, 80\%, 94\% for ER PgR and Her2 respectively.

Conclusion:CNB has proven a valuable and an adequate minimally invasive method for obtaining an accurate representative tissue. The reliability in obtaining HER2 status can be precisely determined however, ER and PgR should be interpreted with caution.
\end{abstract}

Keywords: Core Needle Biopsy; Excision Biopsy; Concordance; Breast Cancer

\section{Introduction}

With the increased prevalence of breast cancer internationally, efficient, cost effective and reliable diagnostic methods are continuously sought. In the emerging era of minimally invasive procedures, coupled by the concordance of both excisional biopsies (EB) with core needle biopsies (CNB), the surgical practice has witnessed a decline in diagnostic surgical excisions [1]. Because of the increased incidence of detected Ductal Carcinoma in Situ (DCIS), fine needle aspiration cytology has become less reliable in obtaining adequate diagnostic samples. On the other hand, the currently widely used CNB in breast lesions has gained popularity over recent years due to the high accuracy in tissue diagnosis obtained from adequate diagnostic samples. Clinicians base their management strategy on the histological diagnosis and hormonal receptors (HR) status in [2]. Chemotherapy treatment, in particular relies on CNB and accurate reporting of HR results [3]. Several studies have demonstrated high concordance rate between CNB and EB in terms of HR status. [2-6] Yet, re-biopsy is strongly indicated in metastatic breast cancer, which may show high discordance between primary and metastatic disease [7].

\section{Materials and Methods}

All patients diagnosed with breast cancer between 2016-2017 were reviewed. The review of the histological data was the essence of this study. Both core needle biopsies (CNB) and excisional biopsies 
(EB) were reviewed. Inclusion criteria included all female patients diagnosed with breast cancer who underwent both diagnostic CNB and EB whose histochemical status was tested in both specimens. The hormonal status of estrogen receptors (ER), progesterone receptors (PgR) and Her2 were evaluated immunohistochemically. Equivocal HER2 status was reassessed using Fluorescence in situ hybridization (FISH) test.

Specimen Handling: The samples were examined by hematoxylin and eosin using 4-microns- thick tissue sections processed from a representative part of the tumor. The tissue was fixed for at least 10 hours in neutral buffered formalin (10\% BV). For the ER, PgR and HER2 immunostaining, specimens were immersed in $10 \%$ neutral buffered formalin within 1 hour of the biopsy or resection procedure and fixed for 6-72 hours. Her2, ER, PgR assay validation was performed using initial test validation included 20 positive and 20 negative cases (FDA-approved Ventana tests). Validation performed by comparing our laboratory's results with a specialist hospital assay that has been appropriately validated. Concordance levels were 95\%. Equivocal cases were not included in Her2 validation.

\section{Reporting and Interpretation Of ER, PgR, HER2 Was Based According To ASCO/CAP Guidelines}

ER and PgR reporting follow that positive test means staining of equal or greater than $1.0 \%$ of tumor cells, whereas a negative test result means staining of less than $1.0 \%$ of tumor cells. Staining is reported as indeterminate if there are problems in sample handling and processing. Scoring was based on the percentage of tumor cells with nuclear staining and staining intensity (weak, moderate or strong); (e.g. ER: 90\% strong, PgR: 50\% weak). As for HER2 immunostaining, positive result is defined as either a (3+) result by immunohistochemistry or a positive Fluorescence In Situ Hybridization (FISH) analysis result.

HER-2 scoring criteria: Her-2 Protein Scoring System: Score 0 (Negative): No staining or faint membrane staining of less than $10 \%$ of tumor cells. Score 1+ (Negative): Weak incomplete membrane staining in more than $10 \%$ of tumor cells. Score $2+$ (Weak Positive): Weak/moderate (thin) complete membrane staining in more than $10 \%$ of tumor cells. Score 3+ (Strong Positive): Strong (thick) complete membrane staining in more than $10 \%$ of tumor cells. Cases reported as equivocal $(2+)$ were tested by FISH. Statistical Analysis was performed using Kappa values.

\section{Results}

112 patients were selected for this study. Seventy-six (67.8\%) patients were excluded due to non-fulfillment of the inclusion criteria. 36 (32.1\%) of both CNB and EB underwent HR analysis in both specimens, and 34 (30.3\%) underwent Her2 testing in CNB and EB.
Types of breast cancer encountered were invasive ductal carcinoma, 67 cases (93\%), invasive lobular carcinoma, 2 cases (2.7\%), mucinous carcinoma, 1 case $(1.3 \%)$, mammary carcinoma with mucinous features, 1 case $(1.3 \%)$, and solid papillary carcinoma, 1 case $(1.3 \%)$.

The diagnosis was initially reported by group of certified pathologists; however, these results were reviewed by a single pathologist for the purpose of this study. ER was positive in 27 (75\%). Thirty-one cases (86\%) were concordant, while five cases (13.8\%) were discordant. Kappa value was 0.67 (Table 1). As for PgR, 22 (61.1\%) cases were positive in the CNB. Twenty-nine (80\%) were concordant with the EB, while 7 (20\%) were discordant. Kappa value was 0.62 (Table 2). HER2 scores were concordant in 32 (94\%) cases. Nine (26.5\%) were positive, while 27 (79.4\%) were reported as negative. Discordance was reported in 2 (6\%). Kappa value is 0.84 (Table 3). Twenty-nine patients received neoadjuvant chemotherapy prior to EB. Three of them had discordant ER results; one patient had both discordant ER and PgR results, and 1 with discordant HER2 result. Thus, the inter-rater agreement between the CNB and EB is 0.67 for ER, 0.62 for PgR and 0.84 for HER2.

Table 1: ER results demonstrated in both CNB and EB samples.

\begin{tabular}{|c|c|c|c|}
\hline \multirow{2}{*}{ CNB } & \multicolumn{3}{|c|}{ ER: Excision } \\
\cline { 2 - 4 } & POS & NEG & Total \\
\hline POS & 23 & 4 & 27 \\
\hline NEG & 1 & 8 & 9 \\
\hline Total & 24 & 12 & 36 \\
\hline concordance 86\% $(31 / 36)$ & \multicolumn{3}{|c}{$\mathrm{k}: 0.67$} \\
\hline
\end{tabular}

Table 2: PgR results demonstrated in both CNB and EB samples.

\begin{tabular}{|c|c|c|c|}
\hline \multirow{2}{*}{ CNB } & \multicolumn{3}{|c|}{ PgR: Excision } \\
\cline { 2 - 4 } & POS & NEG & Total \\
\hline POS & 15 & 7 & 22 \\
\hline NEG & 0 & 14 & 14 \\
\hline Total & 15 & 21 & 36 \\
\hline concordance 80\% $(29 / 36)$ & \multicolumn{3}{|c}{ k:0.62 } \\
\hline
\end{tabular}

Table 3: Her2 results demonstrated in both CNB and EB samples.

\begin{tabular}{|c|c|c|c|}
\hline \multirow{2}{*}{ CNB } & \multicolumn{3}{|c|}{ HER2: Excision } \\
\cline { 2 - 4 } & POS & NEG & Total \\
\hline POS & 7 & 1 & 8 \\
\hline NEG & 1 & 25 & 26 \\
\hline Total & 8 & 26 & 34 \\
\hline concordance $94 \%(32 / 34)$ & \multicolumn{3}{|c}{$\mathrm{k}: 0.84$} \\
\hline
\end{tabular}

\section{Discussion}

Breast cancer continues to be ranked first among females worldwide, the fast leap of expansion in many developing countries have imposed changes in lifestyle has resulted in many alterations 
in disease pattern increasing the prevalence of breast cancer [1]. To be able to establish the histological diagnosis that impacts on treatment outcomes, adequate tissue biopsy is mandatory. Excisional biopsy certainly provides larger tissue samples which allow the pathologist to work with ease in reaching a diagnosis, however, it may necessitate general anesthesia, open surgical procedure and delayed further management. Furthermore, a second procedure may lead to architectural distortion of the surgical field leading to a higher incidence of incomplete excisions. The growing demand for prognostic information in determining the receptor status preoperatively is considered a corner stone in modern multidisciplinary treatment. Given the available numerous improved types of with the efficiency, cost effectivity and the adequate tissue yield, promotes the diagnostic accuracy on HR status assessment [2-6]. The importance of assessing the HR status is well demonstrated in evaluating molecular subtypes, ER, PgR, and HER2 status in breast cancer. Estrogen receptor remains a powerful predictive factor for response to endocrine treatment and long-term outcome. Similarly, HER2 overexpression has been associated with worse prognosis in patients with newly diagnosed breast carcinoma [8]. In some cases where the inherent Ki67 heterogeneity in ER+/HER2- diagnosed breast tumors, distinguishing the molecular subtypes maybe difficult in the CNB specimens. In such cases, reliability on excisional biopsy is recommended [9]. The receptor status is significantly associated with treatment and monitoring in advanced disease.[10] The liberal use of chemotherapy as neoadjuvant treatment by many oncologists was reported to change the tumor profile and baseline receptor status [11]. Imaging guided biopsy has also proven its reliability with acceptable concordance of the receptor status between surgery and ultrasound-guided CNB [12]. In this review, ER concordance rate in our study was $86 \%$. Four patients were found to be positive in CNB and negative in the subsequent excisional only one was patient found to be positive on excision, while there was no reactivity in CNB. Similarly, PgR concordance rate was $80 \%$. The discordance was only in 7 patients who were initially reported as positive in CNB and were negative in EB although the concordance rate of ER and PgR is within the acceptable range, it may falsely miss a significant number of patients. [2-6] For this reason, it is recommended to reevaluate the HR status in surgically excised specimens. This discrepancy can partly be explained by the known effect of neoadjuvant chemotherapy as shown in 5 of our patients. We believe it is due to tumor heterogeneity, which has aslo been documented by other authors. It was also noted that Her2 results demonstrated more significant concordance rate of $94 \%$ this is probably due to the more robust criteria for identifying HER2 immunostaining in addition to the role of FISH in equivocal cases.

\section{Conclusion}

The accuracy of the status in is of paramount importance for both prognosis and treatment. Caution in result interpretation by experienced pathologists may impose significant accuracy and eliminate the false positive results.

\section{Limitation of the Study}

This is a small sample to draw firm conclusions, a larger series is required in future studies.

\section{Conflict of Interest}

The authors declare that there is no conflict of interests.

\section{References}

1. AbdelHadi M, Arida A Linal, Khalifa Amal, Al Haddad H (2017) The impact of Intraoperative Radiotherapy on breast cancer early detection: An Observational Review. Int J Cancer Res Ther 2(3): 1-3.

2. Usami S, Moriya T, Amari M, Suzuki A, Ishida T, et al. (2007) Reliability of prognostic factors in breast carcinoma determined by core needle biopsy. Japanese journal of clinical oncology 37(4): 250-255.

3. Kaufmann M, Hortobagyi G, Goldhirsch A, Scholl S, Makris A, et al. (2006) Recommendations from an international expert panel on the use of neoadjuvant (primary) systemic treatment of operable breast cancer: an update. Journal of Clinical Oncology 24(12): 1940-1949.

4. Cahill R, Walsh D, Landers R, Watson R (2006) Preoperative profiling of symptomatic breast cancer by diagnostic core biopsy. Annals of surgical oncology 13(1): 45-51.

5. Burge C, Chang H, Apple S (2006) Do the histologic features and results of breast cancer biomarker studies differ between core biopsy and surgical excision specimens? The Breast 15(2): 167-172.

6. Cavaliere A, Sidoni A, Scheibel M, Bellezza G, Brachelente G, et al. (2005) Biopathologic profile of breast cancer core biopsy: is it always a valid method? Cancer letters 218(1): 117-121.

7. Lower E, Glass E, Bradley D, Blau R, Heffelfinger S, et al. (2005) Impact of metastatic estrogen receptor and progesterone receptor status on survival. Breast cancer research and treatment 90(1): 65-70.

8. Arnedos M, Nerurkar A, Osin P, A'hern R, Smith I, et al. (2009) Discordance between core needle biopsy (CNB) and excisional biopsy (EB) for estrogen receptor (ER), progesterone receptor (PgR) and HER2 status in early breast cancer (EBC). Annals of oncology 20(12): 19481952.

9. Chen X, Sun L, Mao Y, Zhu S, Wu J, et al. (2013) Preoperative core needle biopsy is accurate in determining molecular subtypes in invasive breast cancer. BMC cancer 13(1): p. 390.

10. Anderson H, Hills M, Zabaglo L, A'hern R, Leary A, et al. (2011) Relationship between estrogen receptor, progesterone receptor, HER-2 and Ki67 expression and efficacy of aromatase inhibitors in advanced breast cancer. Annals of oncology 22(8): 1770-1776.

11. Hirata T, Shimizu C, Yonemori K, Hirakawa A, Kouno T, et al. (2009) Change in the hormone receptor status following administration of neoadjuvant chemotherapy and its impact on the long-term outcome in patients with primary breast cancer. British journal of cancer 101(9): $1529-1536$ 
12. Park Y, Youk J, Son E, Gweon H, Kim J, et al. (2014) Comparison of hormonal receptor and HER2 status between ultrasound-guided 14-gauge core needle biopsy and surgery in breast cancer patients. Ultrasonography 33(3): 206-215.

13. Lee S, Chung M, Quddus M, Steinhoff M, Cady B, et al. (2003) The effect of neoadjuvant chemotherapy on estrogen and progesterone receptor expression and hormone receptor status in breast cancer. The American journal of surgery 186(4): 348-350.

14. Zidan A, Brown J, Peston D, Shousha S (1997) Oestrogen and progesterone receptor assessment in core biopsy specimens of breast carcinoma. Journal of clinical pathology 50(1): 27-29.
15. Layfield L, Saria E, Mooney E, Liu K, Dodge R, et al. (1998) Tissue heterogeneity of immunohistochemically detected estrogen receptor: implications for image analysis quantification. American journal of clinical pathology 110(6): 758-764.

16. Charpin C, Martin P, DeVictor B, Lavaut M, Habib M, et al. (1998) Multiparametric study (SAMBA 200) of estrogen receptor immunocytochemical assay in 400 human breast carcinomas: analysis of estrogen receptor distribution heterogeneity in tissues and correlations with dextran coated charcoal assays and morphological data. Cancer research. 48(6): 1578-1586.

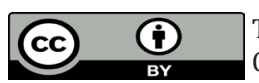

This work is licensed under Creative Commons Attribution 4.0 License

To Submit Your Article Click Here:

Submit Article

DOI: $10.32474 /$ OAJOM.2020.03.000175

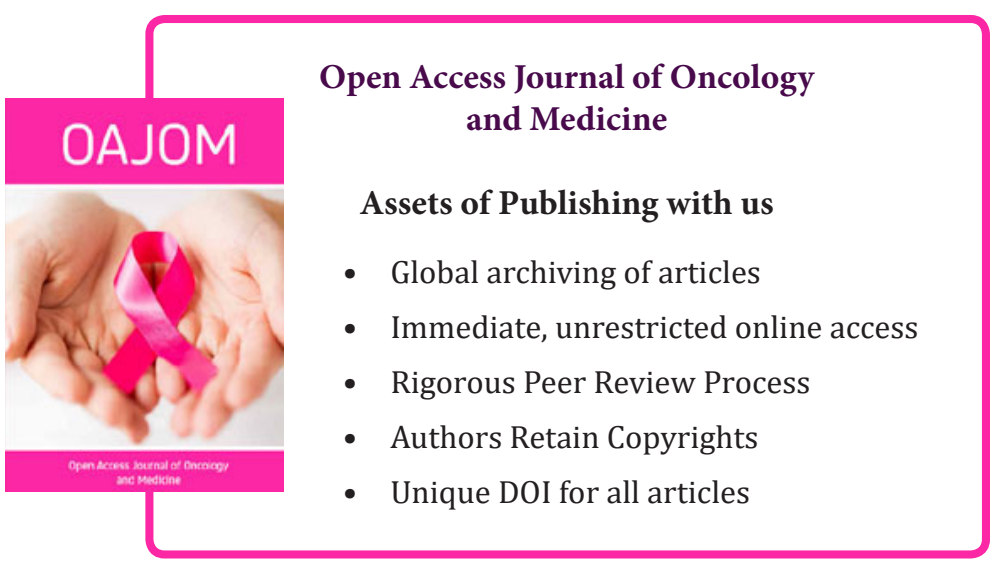

